Supporting Information

\title{
Reactivity of a Platinum Iminoboryl Complex toward Lewis and Brønsted Acids
}

\author{
Holger Braunschweig, ${ }^{*}$ Krzysztof Radacki, Daniela Rais, \\ Achim Schneider and Fabian Seeler \\ Institut für Anorganische Chemie, Bayerische Julius-Maximilians-Universität \\ Würzburg, Am Hubland, D-97074 Würzburg, Germany
}




\section{General considerations}

All manipulations were performed under an inert atmosphere of dry argon using either standard Schlenk-line or glovebox techniques. Benzene and hexane were dried by distillation over potassium and sodium/potassium alloy, respectively; then they were stored under argon over activated molecular sieves. $\mathrm{CH}_{2} \mathrm{Cl}_{2}$ was purified and dried using an $\mathrm{M}$. Braun Solvent Purification System; then it was stored over activated molecular sieves. $\mathrm{CD}_{2} \mathrm{Cl}_{2}$ was dried over molecular sieves and degassed by three freeze-pump-thaw cycles before use. $\mathrm{AlCl}_{3}$ was purified twice by sublimation. NMR spectra were acquired on a Bruker Avance 500 NMR spectrometer. ${ }^{15} \mathrm{~N},{ }^{1} \mathrm{H}$ COSY spectra were recorded on a DRX 300 spectrometer. ${ }^{1} \mathrm{H}$ and ${ }^{13} \mathrm{C}\left\{{ }^{1} \mathrm{H}\right\}$ NMR spectra were referenced to external tetramethylsilane via the residual protio solvent $\left({ }^{1} \mathrm{H}\right)$ or the solvent itself $\left({ }^{13} \mathrm{C}\right) .{ }^{11} \mathrm{~B}\left\{{ }^{1} \mathrm{H}\right\}$ NMR spectra were referenced to external $\mathrm{BF}_{3} \cdot \mathrm{OEt}_{2}$ and ${ }^{31} \mathrm{P}\left\{{ }^{1} \mathrm{H}\right\}$ NMR spectra to $85 \% \mathrm{H}_{3} \mathrm{PO}_{4}$. Microanalyses were performed on an Elementar vario MICRO cube elemental analyzer.

\section{Synthetic procedures and spectroscopic data}

Preparation of trans $-\left[\left(\mathrm{PCy}_{3}\right)_{2} \mathbf{P t}\left\{\mathbf{B N}\left(\mathrm{SiMe}_{3}\right)\left(\mathrm{AlCl}_{3}\right)\right\}(\mathbf{B r})\right](3)$ : A pale yellow solution of trans $-\left[\left(\mathrm{PCy}_{3}\right)_{2} \mathrm{Pt}\left(\mathrm{B} \equiv \mathrm{NSiMe}_{3}\right)(\mathrm{Br})\right](2)(0.103 \mathrm{~g}, 0.110 \mathrm{mmol})$ in dichloromethane $(4 \mathrm{~mL})$ was added to $\mathrm{AlCl}_{3}(0.015 \mathrm{~g}, 0.112 \mathrm{mmol})$. After stirring for $24 \mathrm{~h}$ a green mixture was obtained. The solvent was removed in vacuo; then the residue was extracted with benzene $(2 \mathrm{~mL})$. The insoluble components were removed by filtration and the resulting dark green filtrate was layered with hexane $(4 \mathrm{~mL})$. The solvent was allowed to evaporate slowly to give a gray powder. Subsequent recrystallization from benzene/hexane yielded an analytically pure sample of $\mathbf{3}$ as a pale brown crystalline solid $(0.053 \mathrm{~g}, 45 \%)$. Colorless single crystals of $\mathbf{3}$ were obtained upon slow evaporation of a dichloromethane/hexane solvent mixture.

${ }^{1} \mathrm{H}$ NMR $\left(500 \mathrm{MHz}, \mathrm{CD}_{2} \mathrm{Cl}_{2}, 299.2 \mathrm{~K}\right.$ ): $\delta=2.93$ (br m, 6H, Cy), 2.14-1.22 (m, 60H, Cy), 0.52 (s, 9H, SiMe $) ;{ }^{11} \mathrm{~B}\left\{{ }^{1} \mathrm{H}\right\} \mathrm{NMR}\left(160 \mathrm{MHz}, \mathrm{CD}_{2} \mathrm{Cl}_{2}, 299.2 \mathrm{~K}\right): \delta=46$ (br s); ${ }^{13} \mathrm{C}\left\{{ }^{1} \mathrm{H}\right\}$ NMR $\left(126 \mathrm{MHz}, \mathrm{CD}_{2} \mathrm{Cl}_{2}, 299.2 \mathrm{~K}\right): \delta=36.0\left(\mathrm{vt}, \mathrm{N}=\left|{ }^{1} \mathrm{~J}_{\mathrm{P}-\mathrm{C}}+{ }^{3} \mathrm{~J}_{\mathrm{P}-\mathrm{C}}\right|=23 \mathrm{~Hz}, \mathrm{C}_{1} C y\right)$, 30.2 (s, $\mathrm{C}_{3,5} C y$ ), 27.0 (2 overlapping vt, $\left.\mathrm{N}=\left.\right|^{2} \mathrm{~J}_{\mathrm{P}-\mathrm{C}}+{ }^{4} \mathrm{~J}_{\mathrm{P}-\mathrm{C}} \mid=10 \mathrm{~Hz}, \mathrm{C}_{2,6} C y\right), 26.2\left(\mathrm{~s}, \mathrm{C}_{4} C y\right)$, 3.9 (s, SiMe $)$ ); ${ }^{31} \mathrm{P}\left\{{ }^{1} \mathrm{H}\right\} \mathrm{NMR}\left(202 \mathrm{MHz}, \mathrm{CD}_{2} \mathrm{Cl}_{2}, 299.2 \mathrm{~K}\right): \delta=31.2\left(\mathrm{~s},{ }^{1} \mathrm{~J}_{\mathrm{Pt}-\mathrm{P}}=2135 \mathrm{~Hz}\right)$.

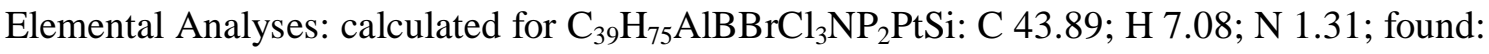
C 44.69; H 7.24; N 1.32 .

Preparation of trans- $\left[\left(\mathrm{PCy}_{3}\right)_{2} \mathbf{P t}\left\{\mathrm{B}(\mathrm{OMe})\left(\mathrm{N}(\mathrm{H}) \mathrm{SiMe}_{3}\right)\right\}(\mathrm{Br})\right](8)$ : A $0.25 \mathrm{M}$ solution of $\mathrm{MeOH}(0.44 \mathrm{~mL}, 0.110 \mathrm{mmol})$ in benzene was added to a solution of trans$\left[\left(\mathrm{PCy}_{3}\right)_{2} \mathrm{Pt}\left(\mathrm{B} \equiv \mathrm{NSiMe}_{3}\right)(\mathrm{Br})\right](2)(0.101 \mathrm{~g}, 0.108 \mathrm{mmol})$ in benzene $(2 \mathrm{~mL})$. After $30 \mathrm{~min}$ the initially clear solution became cloudy. After $3 \mathrm{~h}$ the reaction mixture was layered with hexane $(5 \mathrm{~mL})$ and the solvent mixture was allowed to evaporate slowly. After three days the supernatant liquid was removed by decantation, leaving $\mathbf{8}$ as an analytically pure pale yellow powder $(0.097 \mathrm{~g}, 93 \%)$. Colorless single crystals of $\mathbf{8}$ were obtained upon recrystallization from benzene/hexane. 
${ }^{1} \mathrm{H}$ NMR (500 MHz, $\mathrm{CD}_{2} \mathrm{Cl}_{2}, 295.8 \mathrm{~K}$ ): $\delta=3.85$ (s, 3H, OMe), 2.68 (br m, 6H, Cy), 2.21 (br s, $1 \mathrm{H}, \mathrm{NH}), 2.07-1.23$ (m, 60H, Cy), 0.12 (s, 9H, SiMe $) ;{ }^{11} \mathrm{~B}\left\{{ }^{1} \mathrm{H}\right\}$ NMR $(160 \mathrm{MHz}$, $\left.\mathrm{CD}_{2} \mathrm{Cl}_{2}, 296.1 \mathrm{~K}\right) \delta=30$ (br s); ${ }^{13} \mathrm{C}\left\{{ }^{1} \mathrm{H}\right\} \mathrm{NMR}\left(126 \mathrm{MHz}, \mathrm{CD}_{2} \mathrm{Cl}_{2}, 296.5 \mathrm{~K}\right): \delta=56.9(\mathrm{~s}$, $\mathrm{OMe}$ ), 35.6 (vt, $\left.\mathrm{N}=\left|{ }^{1} \mathrm{~J}_{\mathrm{P}-\mathrm{C}}+{ }^{3} \mathrm{~J}_{\mathrm{P}-\mathrm{C}}\right|=26 \mathrm{~Hz}, \mathrm{C}_{1} C y\right), 31.1\left(\mathrm{~s}, \mathrm{C}_{3,5} C y\right), 29.9\left(\mathrm{~s}, \mathrm{C}_{3,5} C y\right), 27.7$ (2 overlapping vt, $\left.\mathrm{N}=\left.\right|^{2} \mathrm{~J}_{\mathrm{P}-\mathrm{C}}+{ }^{4} \mathrm{~J}_{\mathrm{P}-\mathrm{C}} \mid=11 \mathrm{~Hz}, \mathrm{C}_{2,6} C y\right), 26.9$ (s, $\left.\mathrm{C}_{4} \quad C y\right), 2.3$ (s, SiMe3); ${ }^{31} \mathrm{P}\left\{{ }^{1} \mathrm{H}\right\} \mathrm{NMR}\left(202 \mathrm{MHz}, \mathrm{CD}_{2} \mathrm{Cl}_{2}, 296.0 \mathrm{~K}\right): \delta=25.4\left(\mathrm{~s},{ }^{1} \mathrm{~J}_{\mathrm{Pt}-\mathrm{P}}=2923 \mathrm{~Hz}\right) ;{ }^{15} \mathrm{~N},{ }^{1} \mathrm{H}-\mathrm{COSY}$ $\left({ }^{15} \mathrm{~N}\right.$ : $30 \mathrm{MHz},{ }^{1} \mathrm{H}$ : $\left.300 \mathrm{MHz}, \mathrm{CD}_{2} \mathrm{Cl}_{2}, 296.2 \mathrm{~K}\right)$ : cross-peak between $-292.3\left({ }^{15} \mathrm{~N}\right)$ and 2.21 $\left({ }^{1} \mathrm{H}\right)$. Elemental Analyses: calculated for $\mathrm{C}_{40} \mathrm{H}_{79} \mathrm{BBrNOP}_{2} \mathrm{PtSi}$ : C 49.74, H 8.24, N 1.45; found: C 49.75, H 8.31, N 1.69.

\section{Crystallographic structure determination}

The crystal data of $\mathbf{3}$ and $\mathbf{8}$ were collected at a Bruker X8APEX diffractometer with CCD area detector and multi-layer mirror monochromated $\mathrm{Mo}_{\mathrm{K} \alpha}$ radiation. The structures were solved using direct methods, refined with the Shelx software package (G. Sheldrick, University of Göttingen 1997) and expanded using Fourier techniques. All non-hydrogen atoms were refined anisotropically. Hydrogen atoms were assigned idealized positions and they were included in structure factors calculations.

Crystal data for 3: $\mathrm{C}_{39} \mathrm{H}_{75} \mathrm{AlBBrCl}_{3} \mathrm{NP}_{2} \mathrm{PtSi}, M_{\mathrm{r}}=1067.17$, colorless block, $0.32 \times 0.11 \times 0.10$, orthorhombic space group Pbca, $a=20.2511(5) \AA, \quad b=16.4190(4) \AA, \quad c=28.0348(7) \AA$, $V=9321.6(4) \AA^{3}, Z=8, \rho_{\text {calcd }}=1.521 \mathrm{~g} \cdot \mathrm{cm}^{-3}, \mu=4.181 \mathrm{~mm}^{-1}, F(000)=4336, T=98(2) \mathrm{K}$, $R_{l}=0.0215, w R^{2}=0.0429,9223$ independent reflections $\left[2 \theta \leq 52.16^{\circ}\right]$ and 482 parameters.

Crystal data for 8: $\mathrm{C}_{40} \mathrm{H}_{78} \mathrm{BBrNOP}{ }_{2} \mathrm{PtSi}, \quad M_{\mathrm{r}}=964.87$, colorless plate, $0.160 \times 0.140 \times 0.080$, Monoclinic space group $P 22_{1} / c, \quad a=12.5197(3) \AA, \quad b=13.0247(3) \AA, \quad c=27.1871(6) \AA$, $\beta=96.6100(10)^{\circ}, \quad V=4403.80(18) \AA^{3}, \quad Z=4, \quad \rho_{\text {calcd }}=1.455 \mathrm{~g} \cdot \mathrm{cm}^{-3}, \quad \mu=4.223 \mathrm{~mm}^{-1}$, $F(000)=1980, T=97(2) \mathrm{K}, R_{l}=0.0275, w R^{2}=0.0480,14793$ independent reflections $\left[2 \theta \leq 68.32^{\circ}\right]$ and 434 parameters.

Crystallographic data have been deposited at the Cambridge Crystallographic Data Center as supplementary publication no. CCDC 655184 and 655185 These data can be obtained free of charge from the Cambridge Crystallographic Data Centre via www.ccdc.cam.ac.uk/data_request/cif 